\title{
Strong coupling strategy for fluid-structure interaction problems in supersonic regime via fixed point iteration
}

\author{
Mario A. Storti * , Norberto M. Nigro and Rodrigo R. Paz \\ Centro Internacional de Métodos Computacionales en Ingeniería (CIMEC), \\ INTEC(CONICET-UNL), Güemes 3450, (S3000GLN) \\ Santa Fe, Argentina
}

\begin{abstract}
In this paper some results on the stability of the time integration when solving fluid/structure interaction problems with strong coupling via fixed point iteration strategy are presented. The flow-induced vibration of a flat plate aligned with the flow direction at supersonic Mach number is studied. The precision of different predictor schemes and the influence of the partitioned strong coupling on stability is discussed.
\end{abstract}

Key words: fluid/structure interaction, supersonic flow, parallel computing.

\section{INTRODUCTION}

Multidisciplinary and Multiphysics coupled problems represent nowadays a paradigm when studying/analyzing even more complex phenomena that appear in nature and in new technologies. There exists a great number of problems where different physical processes (or models)

\footnotetext{
* Corresponding author.

Email addresses: mstorti@intec.unl .edu.ar (Mario A. Storti), nnigro@intec.unl.edu. ar (Norberto M. Nigro), rodrigopdintec.unl . edu . ar (Rodrigo R. Paz).

URL: http: / /www. cimec.org. ar (Mario A. Storti).
} 
converge, interacting in a strong or weak fashion (e.g., Acoustics/Noise disturbances in flexible structures, Magneto-Hydrodynamics devices, Micro-Electro-Mechanical devices, ThermoMechanical problems like continuous casting process, Fluid/Structure interaction like wing flutter problem or flow-induced pipe vibrations). In the fluid/structure interaction area, the dynamic interaction between an elastic structure and a compressible fluid has been the subject of intensive investigations in the last years (see References [1-3]). This paper concerns with the numerical integration of this type of problems when they are coupled in a loose or strong manner.

For simple structural problems (like hinged rigid rods with one or two vibrational degrees of freedom) it is possible to combine into a single (simple) formulation the fluid and the structural governing equations (see Reference [4]). In those cases, a fully explicit or fully implicit treatment of the coupled fluid/structure equations is attainable. Nevertheless, for complex/large scale structural problems, the simultaneous solution of the fluid and structure equations using a 'monolithic' scheme may be mathematically unmanageable or its implementation can be a laborious task. Furthermore, the monolithic coupled formulation would change significantly if different fluid and/or structure models were considered.

An efficient alternative is to solve each subproblem in a partitioned procedure where time and space discretization methods could be different. Such a scheme simplifies explicit/implicit integration and it is in favor of the use of different codes specialized on each sub-area. In this work a staggered fluid/structure coupling algorithm is considered. A detailed description of the 'state of the art' in the computational fluid/structure interaction area can be found in works [2,5-7] and the references therein.

Beyond the physical and engineering importance, this problem is interesting from the computational point of view as a paradigm of multiphysics code implementation that reuses preexistent fluid and elastic solvers. The partitioned algorithm is implemented in the PETSc-FEM code (www.cimec.org.ar/petscfem) which is a parallel multi-physics finite element program based on the Message Passing Interface MPI and the Portable Extensible Toolkit for Scientific Computations PETSc. Two instances of the PETSc-FEM code simulate each subproblem and communicate interface forces and displacements via Standard C FIFO files or 'pipes'. The key point in the implementation of this partitioned scheme is the data exchange and synchronization between both parallel processes. These tasks are made in a small external $\mathrm{C}++$ routine.

\section{STRONGLY COUPLED PARTITIONED ALGORITHM VIA FIXED POINT ITER- ATION}

In this section the temporal algorithm that performs the coupling between the structure and the fluid codes is described. It is a fixed point iteration algorithm over the states of both fluid and 
structure systems. Each iteration of the loop is called a 'stage', so if the 'stage loop' converges, then a 'strongly coupled' algorithm is obtained. Hereafter, this algorithm is called 'staged algorithm'. The basic staggered algorithm considered in this work proceeds as follows: (i) transfer the motion of the wet boundary of the solid to the fluid problem, (ii) update the position of the fluid boundary and the bulk fluid mesh accordingly, (iii) advance the fluid system and compute new pressures (and the stress field if compressible Navier-Stokes model is adopted), (iv) convert the new fluid pressure (and stress field) into a structural load, and (v) advance the structural system under the flow loads. Such a staggered procedure, which can be treated as a weakly coupled solution algorithm, can also be equipped with an outer loop in order to assure the convergence of the interaction process. The algorithm can be stated as follow:

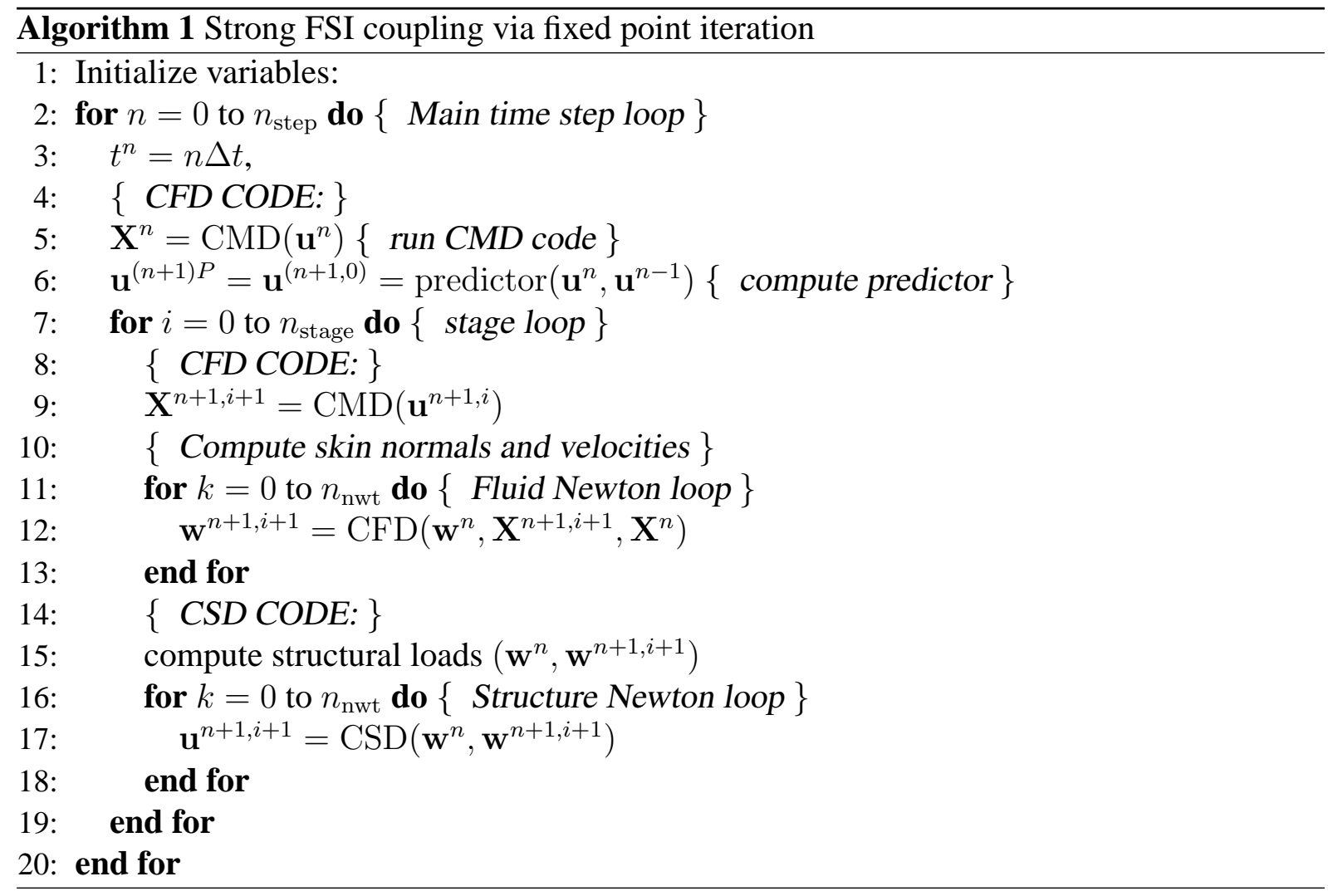

where

$\mathbf{w}^{n}:$ is the fluid state $(\rho, \mathbf{v}, p)$ at time $t^{n}$,

$\mathbf{u}^{n}$ : is the structure state (displacements) at time $t^{n}$,

$\dot{\mathbf{u}}^{n}$ : are structure velocities at time $t^{n}$,

$\mathbf{X}^{n}$ : are fluid mesh node positions at time $t^{n}$,

$n_{\text {step }}:$ is the number of time steps in the simulation,

$n_{\text {stage }}:$ is the number of stages in the coupling scheme

$n_{\text {nwt }}:$ is the number of Newton loops in the nonlinear problem, 
CMD : is intended for Computational Mesh Dynamics,

CSD : for Computational Structure Dynamics,

CFD : for Computational Fluid Dynamics.

\subsection{Notes on the Fluid/Structure Interaction (FSI) algorithm}

- Two codes (CFD and CSD) are running simultaneously. For simplicity, the basic algorithm can be thought as if there were no 'concurrence' between the codes, i.e. at a given time only one of them is running. This can be controlled using 'semaphores' and this is done using MPI 'synchronization messages'.

- The most external loop is over the time steps. Internal to it is the 'stage loop'. 'Weak coupling' is achieved if only one stage is performed (i.e. $n_{\text {stage }}=1$ ). In each stage the fluid is first advanced using the previously computed structure state $\mathbf{u}^{n}$ and the current estimate value $\mathbf{u}^{n+1, i}$. In this way, a new estimate for the fluid state $\mathbf{w}^{n+1, i+1}$ is computed. Next the structure is updated using the forces of the fluid from states $\mathbf{w}^{n}$ and $\mathbf{w}^{n+1, i+1}$. At the first stage, the state $\mathbf{u}^{n+1,0}$ is predicted using a second or higher order approximation (see equation (2)). Inside the stage loop there are Newton loops for each code to solve the non-linearities. In this application the Computational Structure Dynamics (CSD) is linear, so $n_{\mathrm{nwt}}=1$.

- Once the coordinates of the structure are known, the coordinates of the fluid mesh nodes are computed by a 'Computational Mesh Dynamics' code, which is symbolized as

$$
X^{n}=\operatorname{CMD}\left(u^{n}\right)
$$

Even though the CMD may be performed with a general strategy using both nodal reallocation or remeshing, in this paper only the former is adopted, keeping the topology unchanged. Relocation of mesh nodes can be done using an elastic or pseudo-elastic model (see Reference [8]) through a separate PETSc-FEM parallel process (code named MESH-MOVE). For the simple geometry of the example in this paper a simple spine strategy is used.

- The general form of the predictor for the structure state was taken from Reference [2] and can be written as

$$
\mathbf{u}^{(n+1) P}=\alpha_{0} \Delta t \dot{\mathbf{u}}^{n}+\alpha_{1} \Delta t\left(\dot{\mathbf{u}}^{n}-\dot{\mathbf{u}}^{n-1}\right) .
$$

It is at least first order accurate when no predictor is employed and it may be improved to second order using the above predictor with some values for $\alpha_{0}$ and $\alpha_{1}$ according to the problem at hand. To understand how to specify these two parameters a simple two dofs wake oscillator model represented by two second order differential equations as follow

$$
\begin{aligned}
& m_{z} \ddot{z}+c_{z} \dot{z}+k_{z} z=f_{z}(\ddot{y}, \dot{y}, y, t), \\
& m_{y} \ddot{y}+c_{y} \dot{y}+k_{y} y=f_{y}(\ddot{z}, \dot{z}, z, t),
\end{aligned}
$$

with $(m, c, k)$ the mass, the damping and the stiffness parameters for each degree of freedom, $y, z$ representing the structure and the fluid simple model. The forcing terms at the right hand 
side contain the coupling between the two blocks. This coupling may be formulated in terms of the main variables and their two first derivatives, generally velocities and accelerations. If the coupling contains only the main variables, i.e. $f_{z}(y, t)$ and $f_{y}(z, t)$, the predictor with $\alpha_{0}=1$ and $\alpha_{1}=0$ achieves second order accurate in time solutions. If the coupling contains velocities it is necessary to use $\alpha_{1}=1 / 2$ to recover second order in time. In fluid structure interaction problems solved via ALE it is known that the mesh velocity dependent of the fluid-solid interface velocity is incorporated in the formulation, therefore to guarantee second order in time accuracy it is necessary to use $\alpha_{0}=1$ and $\alpha_{1}=1 / 2$ for the predictor.

Note that, if the trapezoidal rule with $\alpha=1 / 2$ is used for both the structure and the fluid and the predictor is chosen with at least second order precision, then the whole algorithm is second order, even if only one stage is performed.

- At the beginning of each fluid stage there is a computation of skin normals and velocities. This is necessary due to the time dependent slip boundary condition for the inviscid case implemented as a constraint (see equation (5)) or also in the case of using a non-slip boundary condition for the viscous case where the fluid interface has the velocity of the moving solid wall, i.e., $\left.\mathbf{v}\right|_{\Gamma_{g}}=\left.\hat{\mathbf{v}}\right|_{\Gamma_{g}}=\left.\dot{\mathbf{u}}\right|_{\Gamma_{g}}$.

\section{DESCRIPTION OF TEST CASE}

The flutter of a flat solid plate aligned with a gas flow at supersonic Mach numbers (see figure (1)) is studied. A uniform fluid at state $\left(\rho_{\infty}, U_{\infty}, p_{\infty}\right)$ flows over an horizontal rigid wall $y=0$ parallel to it. This test case has been studied also in [2].

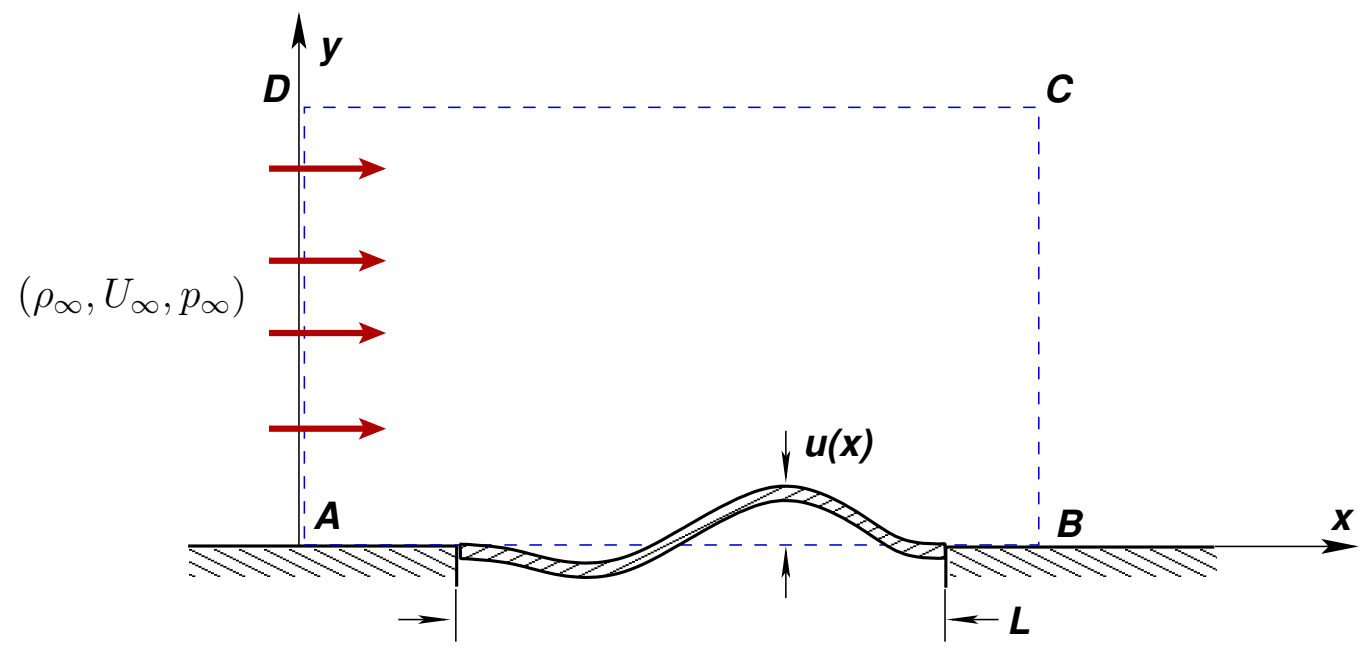

Fig. 1. Description of test

In a certain region of the wall $(0 \leq x \leq L)$ the wall deforms elastically following thin plate 
theory, i.e.

$$
m \ddot{u}+D \frac{\partial^{4} u}{\partial x^{4}}=-\left(p-p_{\infty}\right)+f(x, t),
$$

where $m$ is the mass of the plate per unit area in $\mathrm{Kg} / \mathrm{m}^{2}, D=E \mathrm{t}^{3} / 12\left(1-\nu^{2}\right)$ the bending rigidity of the plate module in $\mathrm{Nm}, E$ is the Young modulus in $\mathrm{Pa}$, $\mathrm{t}$ the plate thickness in $\mathrm{m}, \nu$ the Poisson modulus, $u$ the normal deflection of the plate in m, defined on the region $0 \leq x \leq L$ and null outside this region, $p$ the pressure exerted by the fluid on the plate in $\mathrm{Pa}, f$ is an external force in $\mathrm{N}$ and will be described later. The plate is clamped at both ends, i.e. $u=(\partial u / \partial x)=0$ at $x=0, L$. For the sake of simplicity the fluid occupying the region $y>0$ is inviscid. The compressible Euler model with SUPG stabilization and 'anisotropic shock-capturing' method is considered (see Reference [9]). A slip condition is assumed

$$
\left(\mathbf{v}-\mathbf{v}_{\mathrm{str}}\right) \cdot \hat{\mathbf{n}}=0
$$

on the (curved) wall $y=u(x)$, where

$$
\begin{aligned}
\mathbf{v}_{\text {str }} & =(0, \dot{u}), \\
\hat{\mathbf{n}} & \propto\left(-\frac{\partial u}{\partial x}, 1\right)
\end{aligned}
$$

are the velocity of the plate and its unit normal. Finally, initial conditions for both the fluid and the plate are taken as

$$
\begin{aligned}
u(x, t=0) & =u_{0}(x), \\
\dot{u}(x, t=0) & =\dot{u}_{0}(x), \\
(\rho, \mathbf{v}, p)_{\mathbf{x}, t=0} & =(\rho, \mathbf{v}, p)_{0}, \quad \text { for } y \geq u_{0}(x) .
\end{aligned}
$$

Note that for the fluid pressure load on the plate the free stream fluid pressure is subtracted so that in the absence of any external perturbation $(f \equiv 0)$ the undisturbed flow $(\rho, \mathbf{v}, p)_{x, t} \equiv$ $(\rho, \mathbf{v}, p)_{\infty}$ is a solution of the problem for the initial conditions

$$
\begin{aligned}
u & \equiv 0 \\
\dot{u} & \equiv 0 \\
(\rho, \mathbf{v}, p)_{\mathbf{x}, t=0} & \equiv(\rho, \mathbf{v}, p)_{\infty} .
\end{aligned}
$$

\subsection{Dimensionless parameters}

As the fluid is inviscid, it is determined by the 'adiabatic index' $\gamma=C_{p} / C_{v}=1.4$ for air, and the Mach number $\mathrm{M}_{\infty}=U_{\infty} / c_{\infty}$, where $c_{\infty}$ is the speed of sound $c=\sqrt{\gamma p / \rho}$ for the undisturbed state. 
Another dimensionless parameter can be built by taking the ratio between the characteristic time of the structure which is $T_{\mathrm{str}}=\sqrt{m L^{4} / D}$, and the characteristic time of the fluid $T_{\mathrm{fl}}=L / U_{\infty}$. Then, the dimensionless number $N_{T}$ is defined as the square of the ratio of both characteristic times

$$
N_{T}=\left(\frac{T_{\mathrm{fl}}}{T_{\mathrm{str}}}\right)^{2}=\frac{D}{m L^{2} U_{\infty}^{2}}
$$

Finally, a (dimensionless) number can be formed by taking the ratio between the mass of the fluid being displaced by the structure and the structure mass

$$
N_{M}=\frac{\rho_{\infty} L^{3}}{m L^{2}}=\frac{\rho_{\infty} L}{m}
$$

The same parameters as reported in Reference [2] are considered. In this contribution, flutter was studied near the point $\mathrm{M}_{\infty}=2.27, N_{T}=4.3438 \times 10^{-5}$ and $N_{M}=0.054667$. The flutter region was studied by varying the $\mathrm{M}_{\infty}$ value while keeping $\rho_{\infty}$ and the structure parameters $(\mathrm{m}$, $L, D$ ) constant (so that $N_{M}$ constant and $N_{T} \propto \mathrm{M}_{\infty}{ }^{-2}$ ), and the same approach is taken here. The dimensionless parameters are obtained by choosing the following dimensional values

$$
\begin{aligned}
\rho_{\infty} & =1 \mathrm{Kg} / \mathrm{m}^{3}, \\
p_{\infty} & =1 / \gamma=0.71429 \mathrm{~Pa}, \\
U_{\infty} & =\mathrm{M}_{\infty}, \quad\left(\text { since } c_{\infty}=\sqrt{\gamma p_{\infty} / \rho_{\infty}}=1 \mathrm{~m} / \mathrm{sec}\right), \\
D & =0.031611 \mathrm{Nm}, \\
m & =36.585 \mathrm{Kg} / \mathrm{m}^{2}, \\
L & =2 \mathrm{~m} .
\end{aligned}
$$

\subsection{Houbolt's model}

In this section the linear flutter instability by means of the modal analysis is studied. First, the 'Houbolt approximation' (see Reference [10]) is assumed for the fluid,

$$
\begin{aligned}
p-p_{\infty} & =C_{x} \frac{\partial u}{\partial x}+C_{t} \frac{\partial u}{\partial t}, \\
C_{x} & =\frac{\rho_{\infty} U_{\infty}^{2}}{\sqrt{\mathrm{M}_{\infty}{ }^{2}-1}}, \\
C_{t} & =\frac{\rho_{\infty} U_{\infty}\left(\mathrm{M}_{\infty}{ }^{2}-2\right)}{\left(\mathrm{M}_{\infty}{ }^{2}-1\right)^{3 / 2}} .
\end{aligned}
$$


With this approximation the governing equation for plate deflection (4) becomes

$$
m \ddot{u}+D \frac{\partial^{4} u}{\partial x^{4}}=-C_{x} \frac{\partial u}{\partial x}-C_{t} \frac{\partial u}{\partial t} .
$$

\subsubsection{Plane wave analysis}

If an infinite plate is considered, a plane wave analysis may shed some light on the mechanism that leads to a flutter behavior. Then, let consider plane waves of the form

$$
u(x, t)=\operatorname{Re}\left\{\hat{u} \mathrm{e}^{i(k x-\omega t)}\right\} .
$$

Replacing (14) in (13) an implicit “dispersion law" $\omega=\omega(k)$ it is obtained

$$
-\omega^{2} m+D k^{4}=i C_{t} \omega-i k C_{x}
$$

Regarding the last equation instability (flutter) occurs whenever $\operatorname{Im}\{\omega\}>0$. Note that lowering the mass ratio parameter $N_{M}$ while keeping $N_{T}$ and $\mathrm{M}_{\infty}$ constant, it is equivalent to scaling the fluid terms on the the right hand side of equation (12) by this factor. When there is no fluid $\left(N_{M} \rightarrow 0\right)$ the dispersion law simply reduces to

$$
\omega_{0}= \pm \sqrt{\frac{D}{m}} k^{2} .
$$

As expected, the eigenvalues are real, meaning neither damping nor amplification of the waves. The positive (negative) sign corresponds to right-going (left-going) waves, i.e. waves that run in the same (opposed) direction as the fluid. The subscript 0 indicates that this dispersion law is valid in the absence of fluid. Now assume that $N_{M}$ is small enough so that the right hand side of equation (15) is a small perturbation to the terms in the left hand side. Then a first order expansion of the left hand side with respect to $\omega$ around $\omega_{0}$ can be done

$$
-2 m \omega_{0} \delta \omega=i C_{t} \omega_{0}-i k C_{x}
$$

so that

$$
\omega \approx \omega_{0}-i \frac{C_{t}}{2 m} \pm i \frac{C_{x}}{2 k \sqrt{m D}} .
$$

From this equation it is clear that the temporal term (the second one in the Houbolt approximation (12)) has a stabilizing effect (negative imaginary part), while the spatial term has a damping effect for left-going waves and destabilizing effect for right-going ones, i.e. for those that run in the same direction as the fluid. Flutter occurs when the destabilizing term is strong enough so as to overcome the stabilizing temporal term. 
More physical insight is obtained analyzing the work that is done by the fluid onto the plate. If a plane wave given by equation (14) is considered in its equivalent real form

$$
u(x, t)=|\hat{u}| \cos (k x-\omega t+\varphi),
$$

with $\hat{u}=|\hat{u}| \mathrm{e}^{i \varphi}$, then the instantaneous vertical velocity and pressure are

$$
\begin{aligned}
v & =\frac{\partial u}{\partial t}=\omega|\hat{u}| \sin (k x-\omega t+\varphi), \\
p-p_{\infty} & =\left(-C_{x} k+C_{t} \omega\right)|\hat{u}| \sin (k x-\omega t+\varphi) .
\end{aligned}
$$

The instantaneous work done by the fluid onto the plate, averaged on a wave length $\lambda=2 \pi / k$ is

$$
\begin{aligned}
\dot{W} & =-\int_{0}^{\lambda} p v \mathrm{~d} x, \\
& =\omega\left(C_{x} k-C_{t} \omega\right)|\hat{u}|^{2} \frac{\lambda}{2} .
\end{aligned}
$$

A positive work means that the structure is absorbing energy from the fluid, and then has a destabilizing effect while the opposite means dissipation of the structural wave energy into the fluid. It can be seen (again) from equation (21) that the temporal term has always a stabilizing effect, while the spatial term has a destabilizing effect when $\operatorname{sign}(\omega) \operatorname{sign}(k)>0$, i.e. for rightgoing waves (traveling in the same sense of the fluid).

Note that at the basis of the destabilizing effect is the fact that the spatial term in the Houbolt approximation produces a pressure perturbation field that is non-symmetric with respect to the crest of the waves, i.e. before the crest $((\mathrm{d} u / \mathrm{d} x)>0) p-p_{\text {ref }}>0$ whereas after the crest $((\mathrm{d} u / \mathrm{d} x)<0) p-p_{\text {ref }}<0$. In inviscid subsonic flow the pressure perturbation field would be symmetric.

\subsubsection{Galerkin model for the finite length plate}

The plate normal displacement is expanded in a global basis using

$$
\begin{aligned}
u(x) & =\sum_{k=1}^{N} a_{k} \psi_{k}(x), \\
\psi_{k}(x) & =\frac{4 x(L-x)}{L^{2}} \sin (k \pi x / L) .
\end{aligned}
$$

These basis functions satisfy the essential boundary conditions for the plate equation $u=$ $(\partial u / \partial x)=0$ at $x=0, L$. Replacing the Houbolt approximation in equation (4), using Galerkin method and integrating by parts as needed, the following matrix equation is obtained

$$
\mathbf{M a ̈}+\mathbf{K a}+\mathbf{H}_{x} \mathbf{a}+\mathbf{H}_{t} \dot{\mathbf{a}}=0
$$


where

$$
\begin{aligned}
M_{j k} & =\int_{0}^{L} m \psi_{j}(x) \psi_{k}(x) \mathrm{d} x, \\
K_{j k} & =\int_{0}^{L} D \psi_{j}^{\prime \prime}(x) \psi_{k}^{\prime \prime}(x) \mathrm{d} x, \\
H_{x, j k} & =\int_{0}^{L} C_{x} \psi_{j}(x) \psi_{k}^{\prime}(x) \mathrm{d} x, \\
H_{t, j k} & =\int_{0}^{L} C_{t} \psi_{j}(x) \psi_{k}(x) \mathrm{d} x .
\end{aligned}
$$

Solution of these system of ODE's can be found by standard operational methods by replacing $\mathbf{a}(t)$ by the ansatz

$$
\mathbf{a}(t)=\hat{\mathbf{a}} \mathrm{e}^{\lambda t}
$$

leading to the eigenvalue equation

$$
\left(\lambda^{2} \mathbf{M}+\lambda \mathbf{H}_{t}+\mathbf{K}+\mathbf{H}_{x}\right) \hat{\mathbf{a}}=0 .
$$

Flutter is detected whenever some eigenvalue $\lambda$ has a positive real part.

\subsubsection{Numerical solution details}

- The series (22) are truncated at a certain number of terms $N$. Usually $N=10$ or 20.

- Matrix entries for $\mathbf{M}, \mathbf{K}, \mathbf{H}_{t}$ and $\mathbf{H}_{x}$ are computed by approximating derivatives with second order finite differences and integrating with a second order rule.

- The quadratic eigenvalue problem of size $N$ is solved by converting it to a linear eigenvalue problem of size $2 N$ (and then finding eigenvalues and eigenvectors).

\subsubsection{Results}

Using for instance the values described in (11) with $N=20$ terms in the series and 5000 intervals for computing the matrix coefficients integrals, and varying Mach from 1.8 to 3 the results shown in figure (2) are obtained. For $\mathrm{M}_{\infty}<\mathrm{M}_{\mathrm{cr}}=2.265$, all the eigenvalues have negative real part being stable. For $\mathrm{M}_{\infty}>\mathrm{M}_{\mathrm{cr}}=2.265$ there are two complex conjugate roots with positive real part. In figure (2) the real and imaginary part of the unstable mode are plotted. For $\mathrm{M}_{\infty}<\mathrm{M}_{\mathrm{cr}}=2.265$ the eigenvalue with the lower frequency was taken as a continuation of the flutter mode. It was checked that for $\mathrm{M}_{\infty}<\mathrm{M}_{\mathrm{cr}}=2.265$ the plate does positive power on the fluid whereas for $\mathrm{M}_{\infty}>\mathrm{M}_{\mathrm{cr}}=2.265$ the converse is true. The instantaneous power done by the plate on the fluid is

$$
P=\int_{x=0}^{L} p \dot{u} \mathrm{~d} x
$$




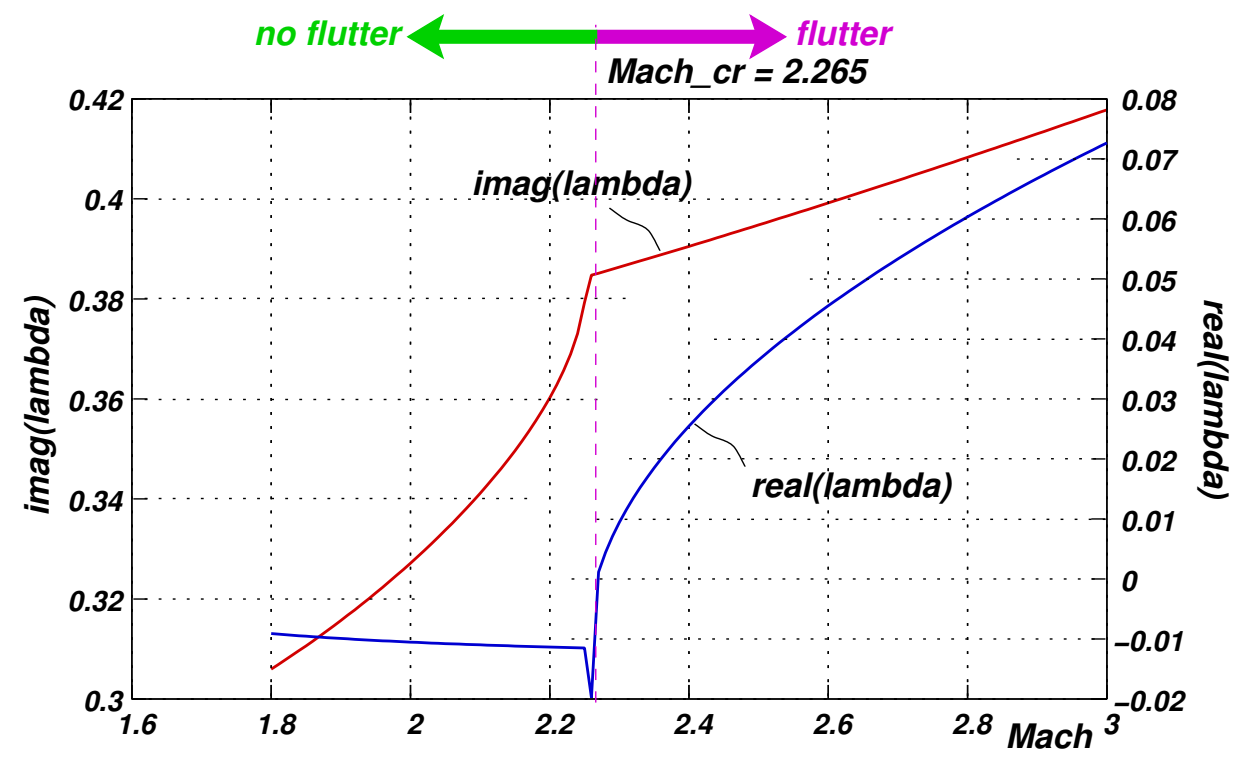

Fig. 2. Lowest frequency mode for test case.

In figures (3) to (5) the form of the plate deflection of the flutter mode for Mach 2.22, 2.27 and 2.35 is observed. For each Mach: plate deflection, fluid pressure on the plate and power that is being done by the plate on the fluid $(p \dot{u})$ are shown.

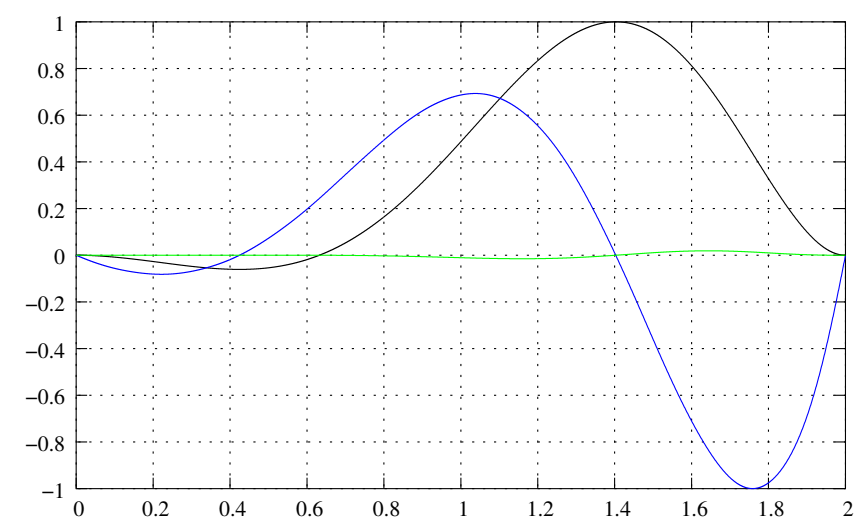

Fig. 3. Mach 2.2, phase 0. Black= plate deflection, blue=pressure, green=power. Quantities normalized (not to scale).

\subsubsection{Flutter region}

A large number of flutter computations in the space $N_{M}, N_{T}, \mathrm{M}_{\infty}$ were performed in order to determine the flutter region. A grid of $20 \times 20$ points in the region $\left[0.001 \leq N_{M} \leq 0.1\right] \times\left[10^{-5} \leq\right.$ $\left.N_{T} \mathrm{M}_{\infty}{ }^{2} \leq 10^{-3}\right]$ was scanned. For each point in the grid instability is scanned in the Mach 


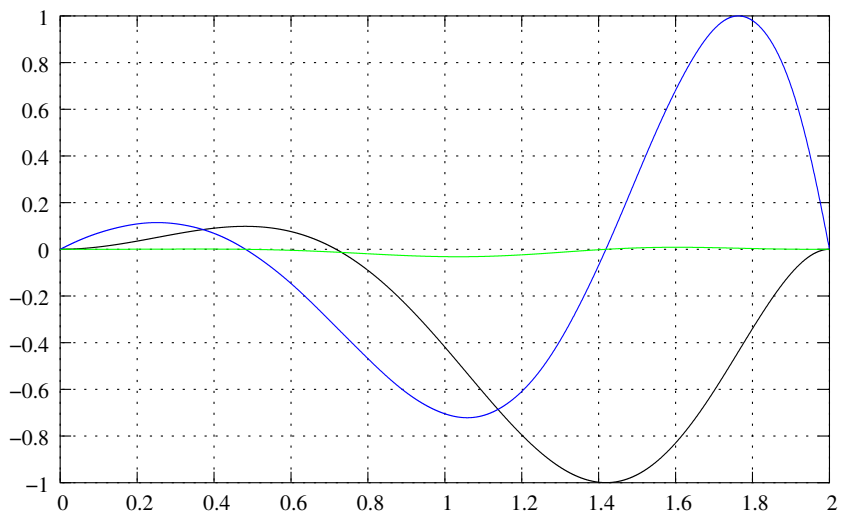

Fig. 4. Mach 2.27, phase 0

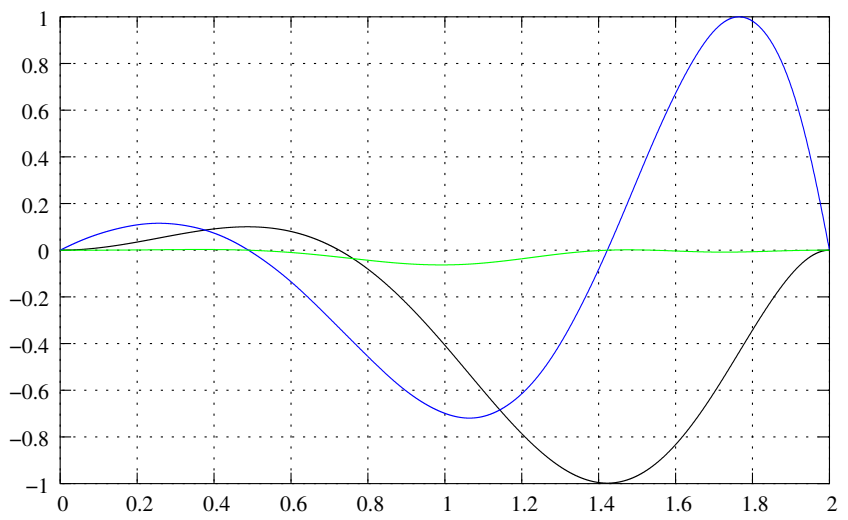

Fig. 5. Mach 2.35, phase 0

region $1.8 \leq \mathrm{M}_{\infty} \leq 3$. The flutter region has the following form:

$$
\begin{aligned}
& \frac{N_{M}}{N_{T} \mathrm{M}_{\infty}^{2}}<200 \text { no flutter for any Mach number, } \\
& \frac{N_{M}}{N_{T} \mathrm{M}_{\infty}^{2}}>300 \text { flutter for the lowest Mach number considered }\left(M_{\infty} \geq 1.8\right) .
\end{aligned}
$$

In the intermediate region flutter is produced. This suggests that flutter is highly correlated to quantity

$$
\frac{N_{M}}{N_{t} \mathrm{M}_{\infty}{ }^{2}}=\frac{\rho_{\infty} L^{3} c_{\infty}}{D}
$$

which happens to be independent of the density of the plate.

A simple model presented in Reference [4] draws a similar conclusion. The explanation is as follows. In that reference, the term proportional to $(\partial u / \partial t)$ is neglected. This is true if the characteristic times of the structure are much lower than those of the fluid, i.e. $N_{T} \ll 1$. This is a valid assumption because of all points in the grid located in the region $N_{T}<10^{-3}$ are 
considered. But if the temporal term in the Houbolt approximation is neglected the characteristic equation can be written in the form

$$
\operatorname{det}\left(\bar{\lambda}^{2} \overline{\mathbf{M}}+\mathbf{K}+\mathbf{H}_{x}\right)=0
$$

where

$$
\begin{aligned}
\bar{\lambda} & =\sqrt{m} \lambda, \\
\overline{\mathbf{M}} & =\frac{1}{\sqrt{m}} \mathbf{M} .
\end{aligned}
$$

As now the coefficients in $\overline{\mathbf{M}}, \mathbf{K}, \mathbf{H}_{x}$ do not depend on $m$, neither do the eigenvalues of equation (30), and then by (31) the $\lambda$ eigenvalues are of the form

$$
\lambda_{j}=\frac{\bar{\lambda}_{j}}{\sqrt{m}}
$$

with $\bar{\lambda}$ not depending on $m$. This means that the sign of the real part of the $\lambda$ is independent of $m$.

\subsection{FSI code results}

The aeroelastic problem defined above was modeled with the strongly coupled partitioned algorithm described in section $\S 2$ with a mesh of 12800 quadrilateral elements for the fluid and 5120 for the plate. As the flow is supersonic only a small entry section of $1 / 8 L$ upstream the plate and $1 / 3 L$ downstream is considered. The vertical size of the computational domain was chosen as $0.8 L$. It is assured that no reflection from the upper boundary affects the plate itself when considering these sizes for the fluid domain.

\subsubsection{Determination of flutter region}

This section presents some results obtained with PETSc-FEM code using the weak coupling between fluid and structure, i.e. $n_{\text {stage }}=1$. The physical characteristics of the plate are the same as in previous section. In order to find (numerically) the critical Mach number for this problem a sweep in the Mach number in the range of 1.8 to 3.2 was done. Results for some Mach numbers can be seen in figures (6) to (11). In these plots the time evolution of displacements of several points distributed along the skin plate are shown. The fluid density field and the structure displacement at Mach=3.2 (flutter region) for a given time step is shown in figures (12) and (13).

For Mach numbers below the $\mathrm{M}_{\mathrm{cr}}$, figures (6) to (8), the maximum plate displacement grows until the forces exerted by the fluid dump the plate displacements. The time needed to reduce the response a given factor (30\% for instance) grows with the Mach number. For Mach numbers 


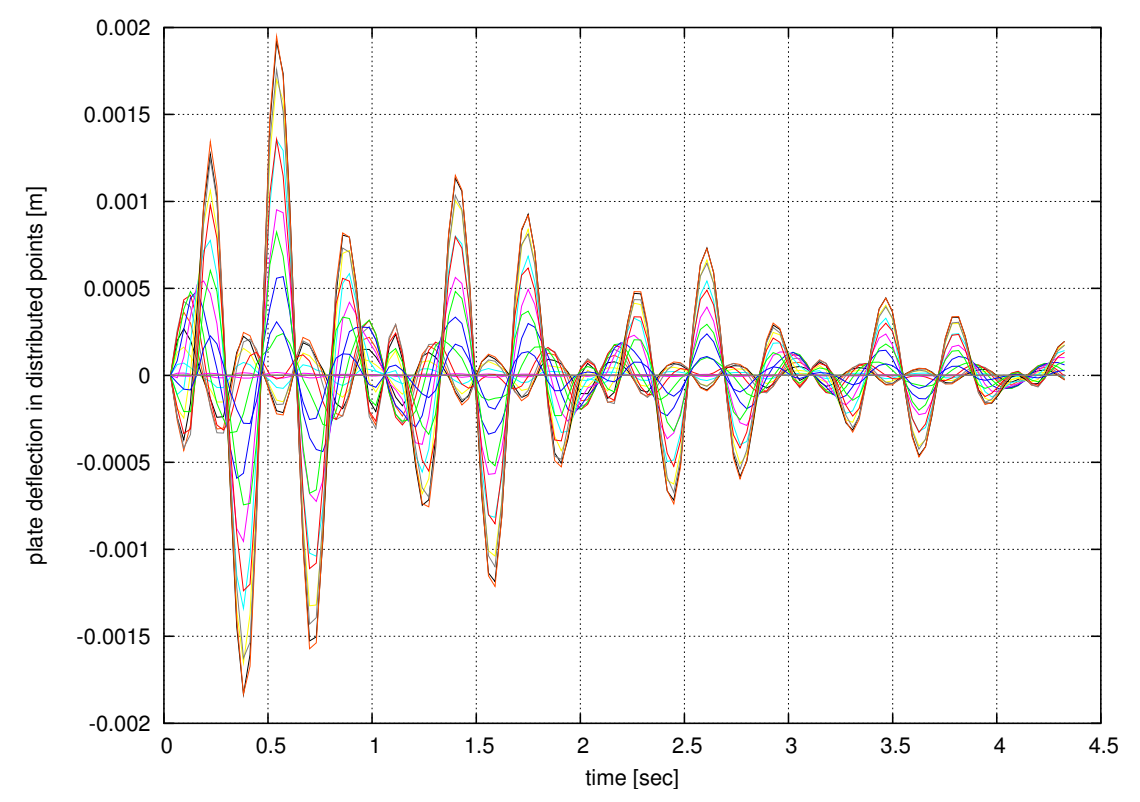

Fig. 6. Plate deflection in distributed points along plate at $\mathrm{M}=1.8$

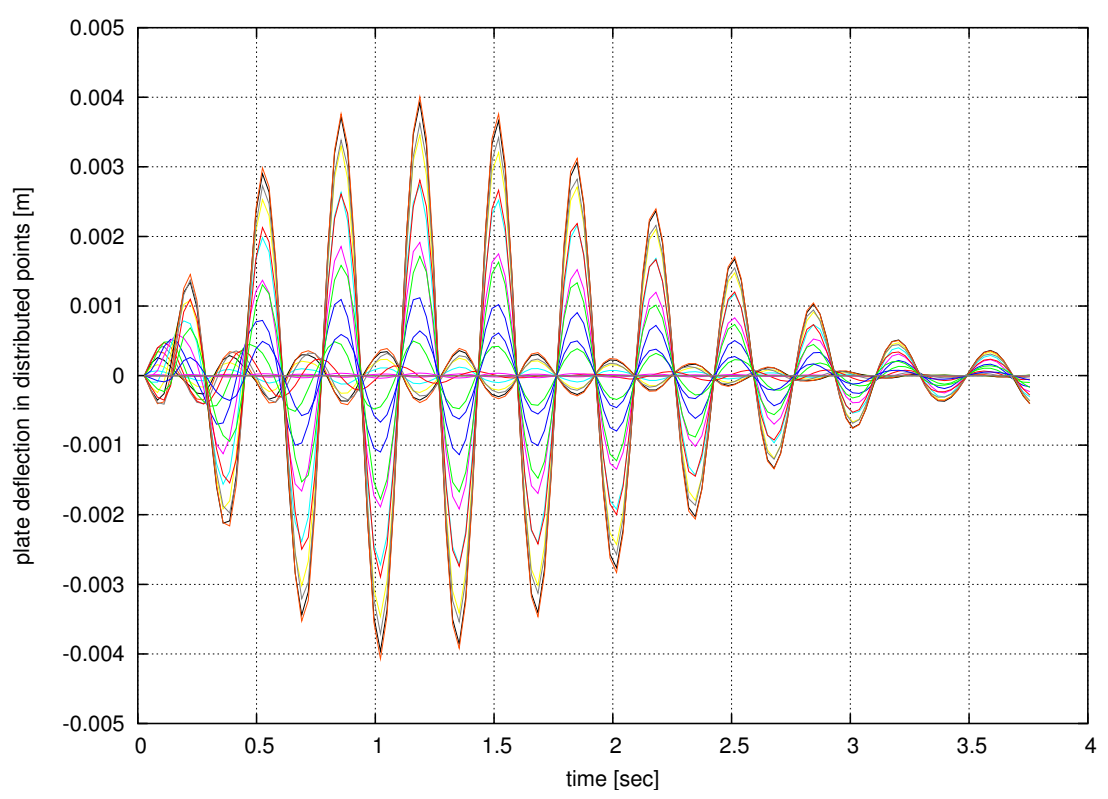

Fig. 7. Plate deflection in distributed points along plate at $\mathrm{M}=2.225$

near the $M_{c r}$, figure (9), the maximum amplitude grows slightly. The flutter mode is triggered at this point. For Mach numbers above the $\mathrm{M}_{\mathrm{cr}}$, figures (10), (11), (12) and (13), the fluid forces cannot damp the structure response and displacements grow without limit in a unstable fashion according to the theory. 


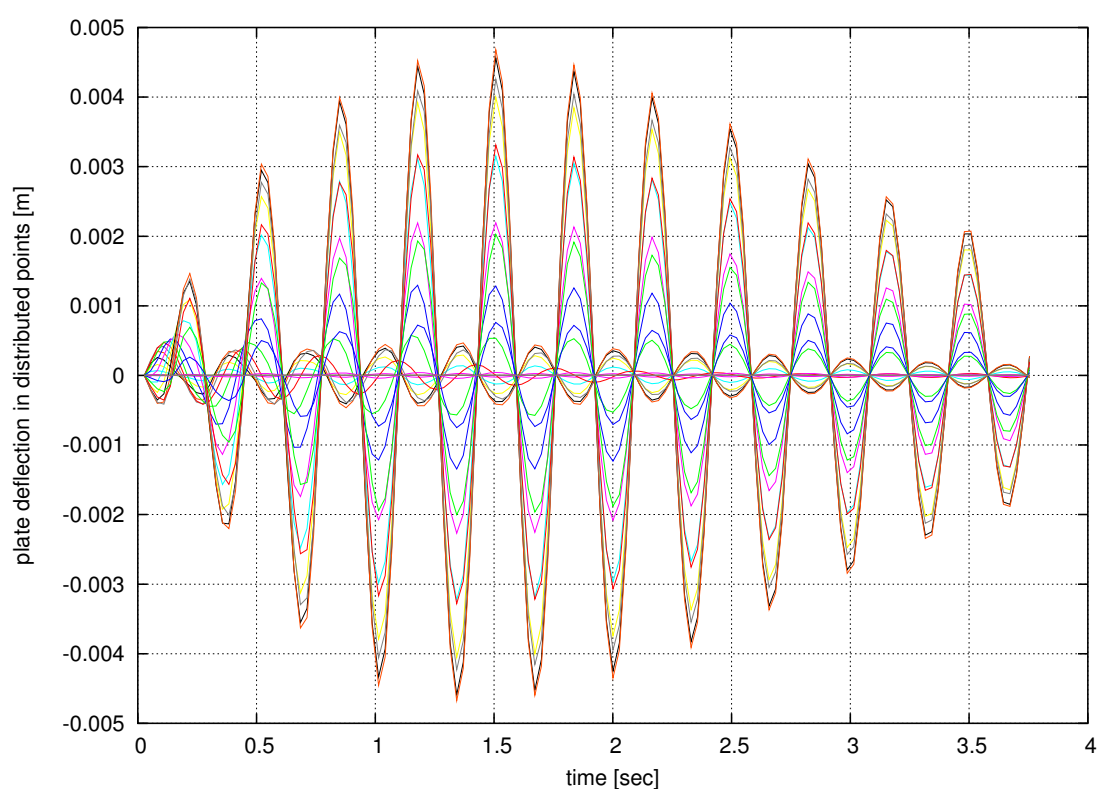

Fig. 8. Plate deflection in distributed points along plate at $\mathrm{M}=2.25$

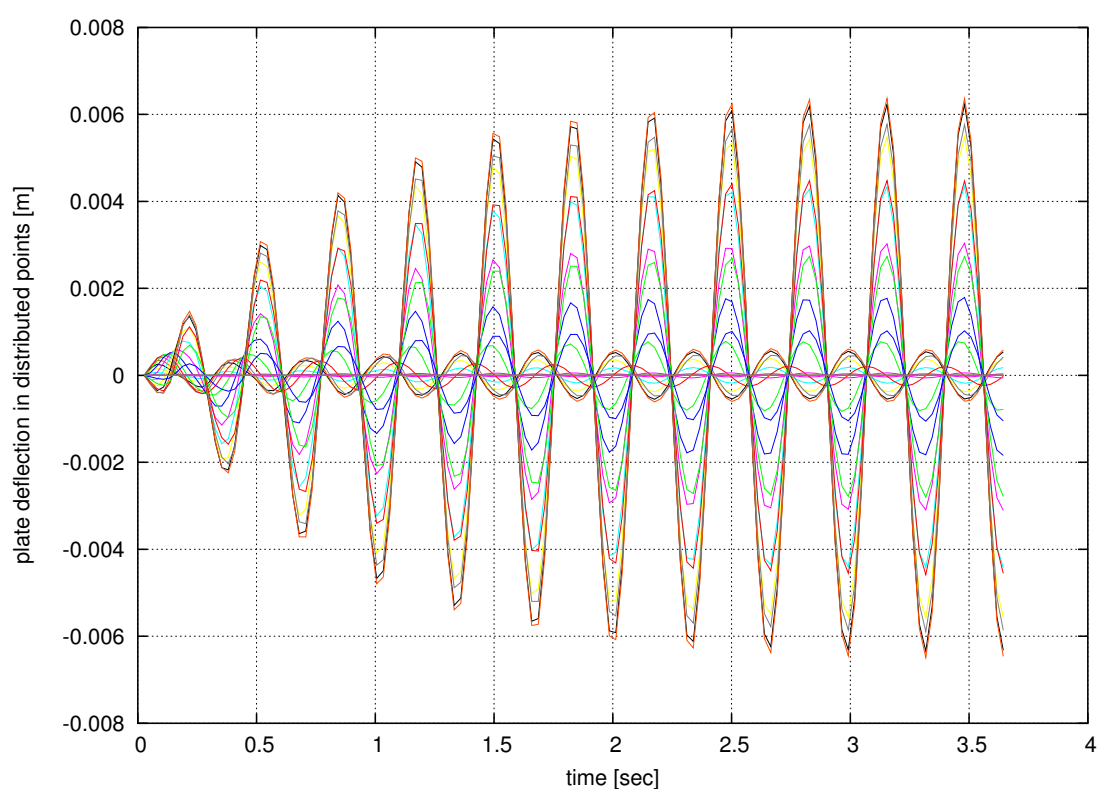

Fig. 9. Plate deflection in distributed points along plate at $M=2.275$

\subsubsection{Time accuracy}

If the stage loop converges, i.e. $(\mathbf{u}, \mathbf{w})^{n+1, i} \rightarrow(\mathbf{u}, \mathbf{w})^{n+1, *}$, then it can be shown that the limit states $(\mathbf{u}, \mathbf{w})^{n+1, *}$ satisfy the fully implicit, strong coupled equations. The main effect of the staged algorithm is to have a strong coupling and then, enhanced stability, regardless the time 


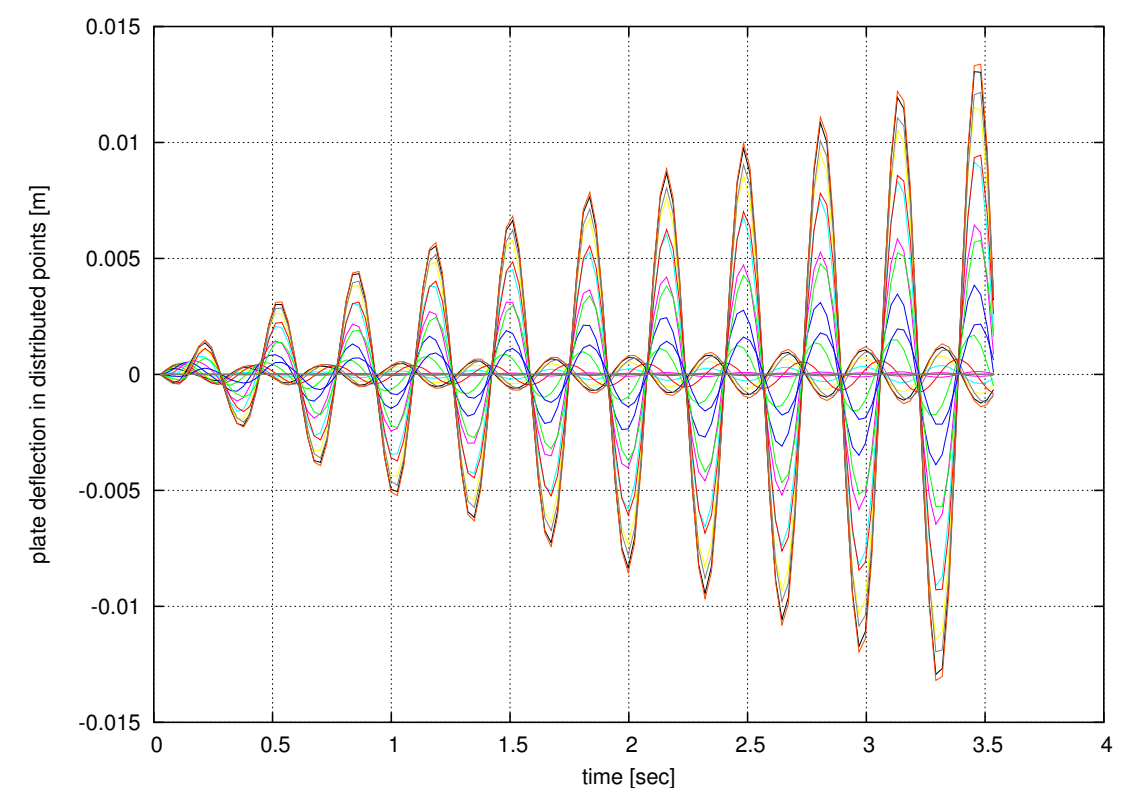

Fig. 10. Plate deflection in distributed points along plate at $M=2.3$

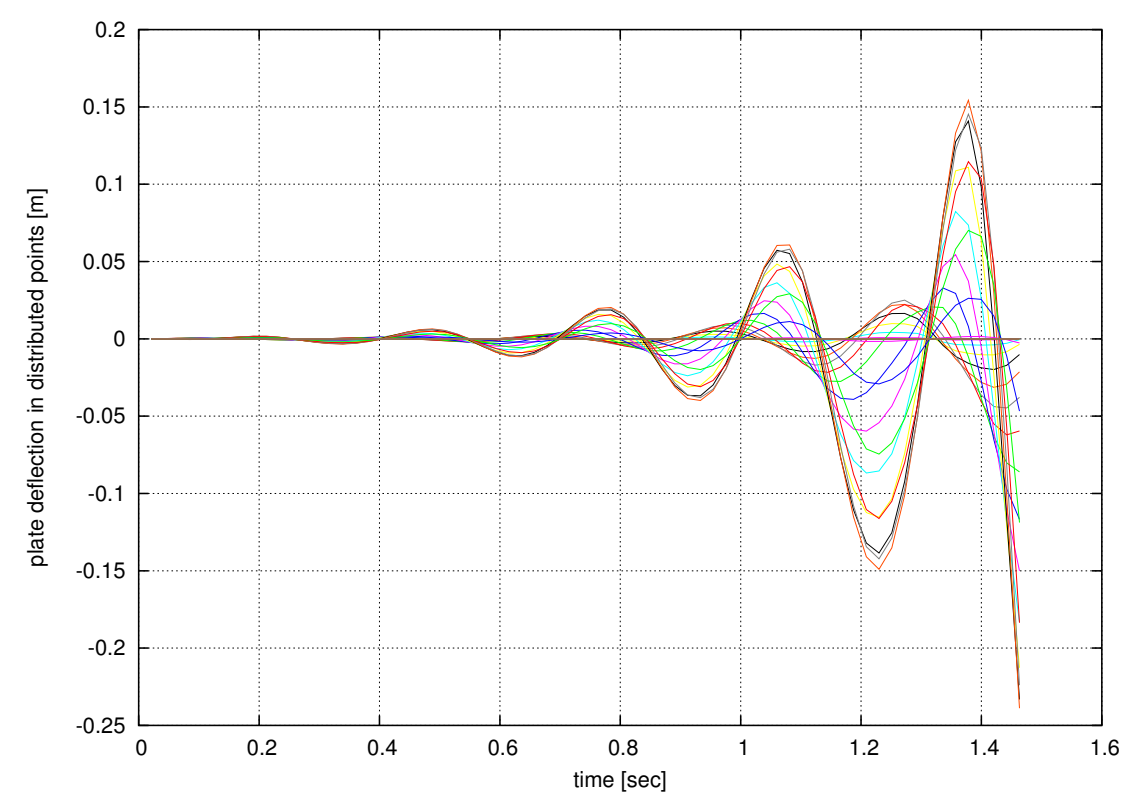

Fig. 11. Plate deflection in distributed points along plate at $\mathrm{M}=3.2$

accuracy, i.e., second or higher order temporal schemes can be achieved with a non-staged weak algorithm, while a strong coupled staged algorithm not necessarily have high order accuracy. In figure (14) the error obtained after the simulation of a certain fixed amount of time $t_{0}$ and increasing time refinement is shown. The exact solution is estimated through a Richardson extrapolation with the two more refined simulations for the more accurate scheme $(\alpha=0.5)$. 


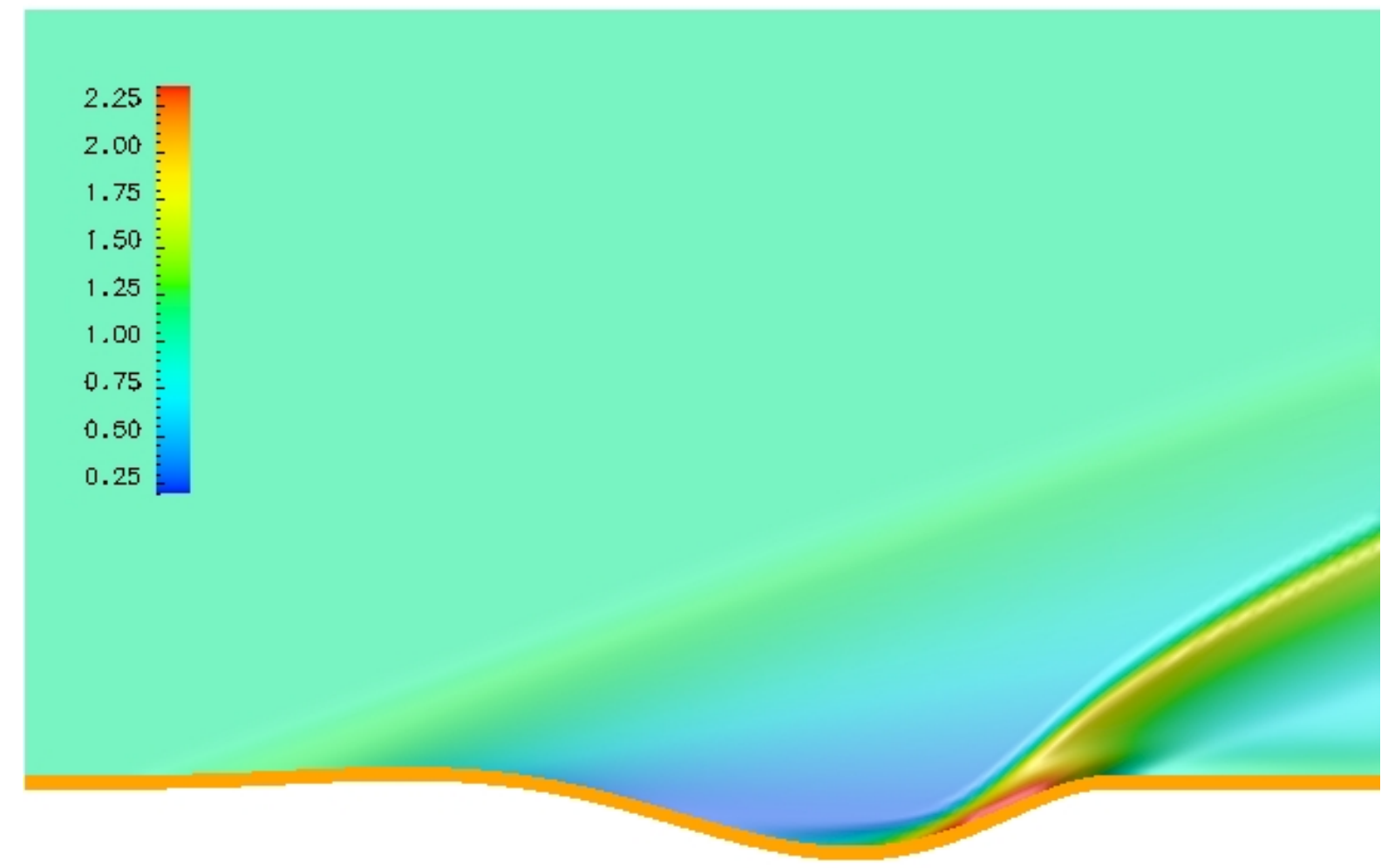

Step 285, time 1.46575 secs, color=density

Fig. 12. Fluid and structure fields at $\mathrm{M}=3.2$

The error at $t_{0}$ is evaluated for a certain number of different $\Delta t$ values. It can be see that for $\alpha=$ 0.6 (the parameter of the trapezoidal rule in both the structure and the fluid) the convergence curve has initially second order slope, but for $\Delta t$ small enough this order is lost. This is typical when the error has mixed first and second order terms, for instance $E \propto c \Delta t+c^{\prime} \Delta t^{2}$. For high $\Delta t$ the second order term rules and a second order convergence is perceived. However, as the time step is diminished, at a certain point the first term rules and the slope switches to first order. For $\alpha=0.5$ the curve is $O\left(\Delta t^{2}\right)$ on the whole studied range of $\Delta t$. When using $\alpha=0.5$ with no predictor (equation (2)), a second order convergence is still obtained, but the convergence slightly slows down in the very last segment. Note that in this case the scheme is second order, except for the fluid-structure interaction. As the interaction is weak, perhaps the first order convergence can be observed for smaller time steps. 


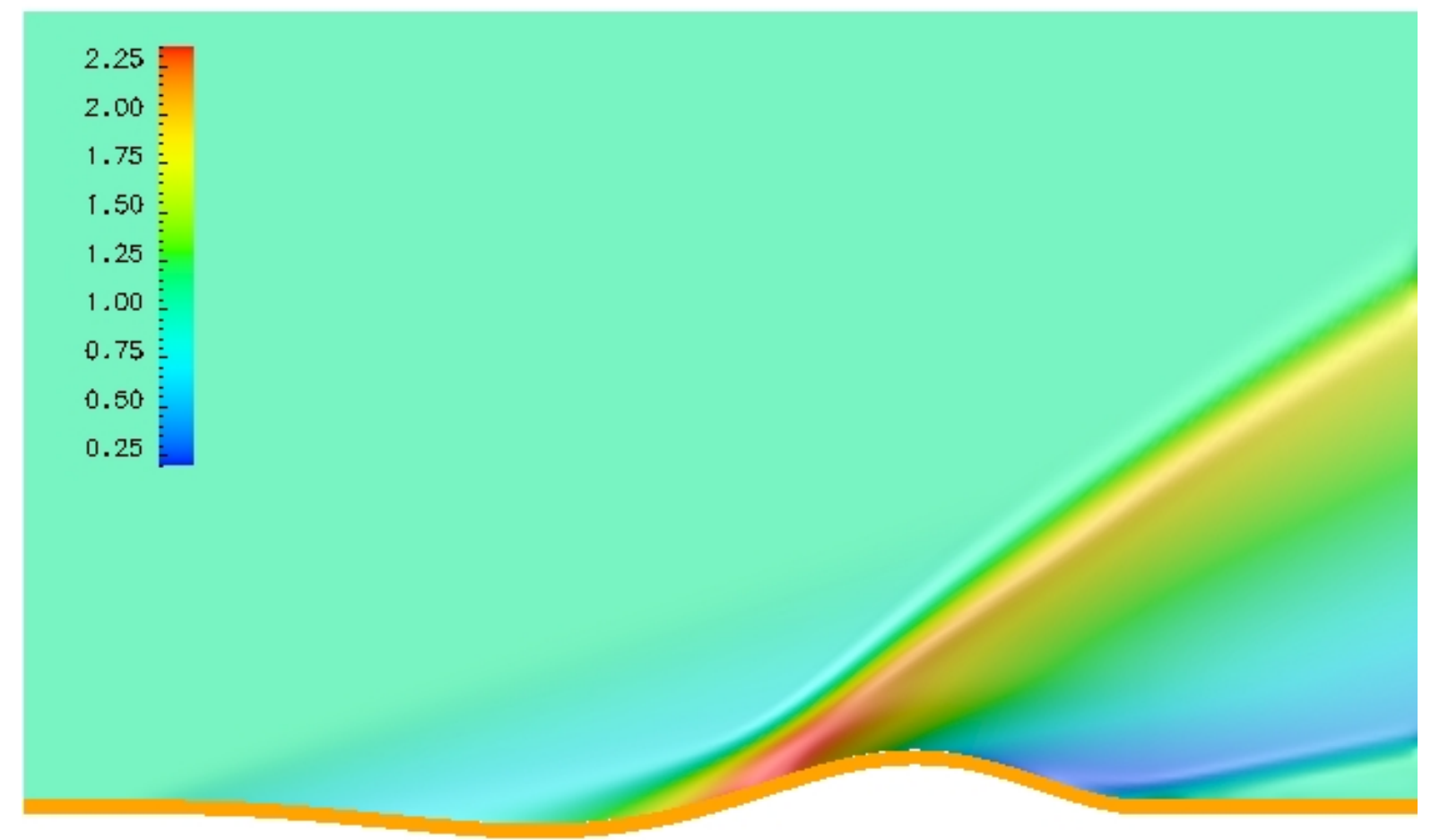

Step 327, time 1.68176 secs, color=density

Fig. 13. Fluid and structure fields at $\mathrm{M}=3.2$

\subsubsection{Convergence of stage loop}

The convergence of the stage loops has been assessed by running the test case over 20 time steps and performing 10 stages at each time step. In figure (15) the convergence of the fluid state (i.e. $\left\|\mathbf{u}^{n+1, i+1}-\mathbf{u}^{n+1, i}\right\|$ ) for all the time steps (convergence curves of the time steps are concatenated) is shown. Analogously, the convergence of the structure is plotted in figure (16). The average convergence is one order of magnitude per stage or higher, suggesting that for such a situation a small $n_{\text {stage }}(2$ or 3 ) would be enough.

\subsubsection{Stability of the staged algorithm}

The following numerical test allows to evaluate the stability of the staged algorithm presented in section $\S 2$. The example is similar to the aeroelastic test case presented in section $\S 3$ with some different parameters for the plate in order to produce larger plate deformations and stronger instabilities. Some parameters are similar to those presented in equation (11). Here, only the 


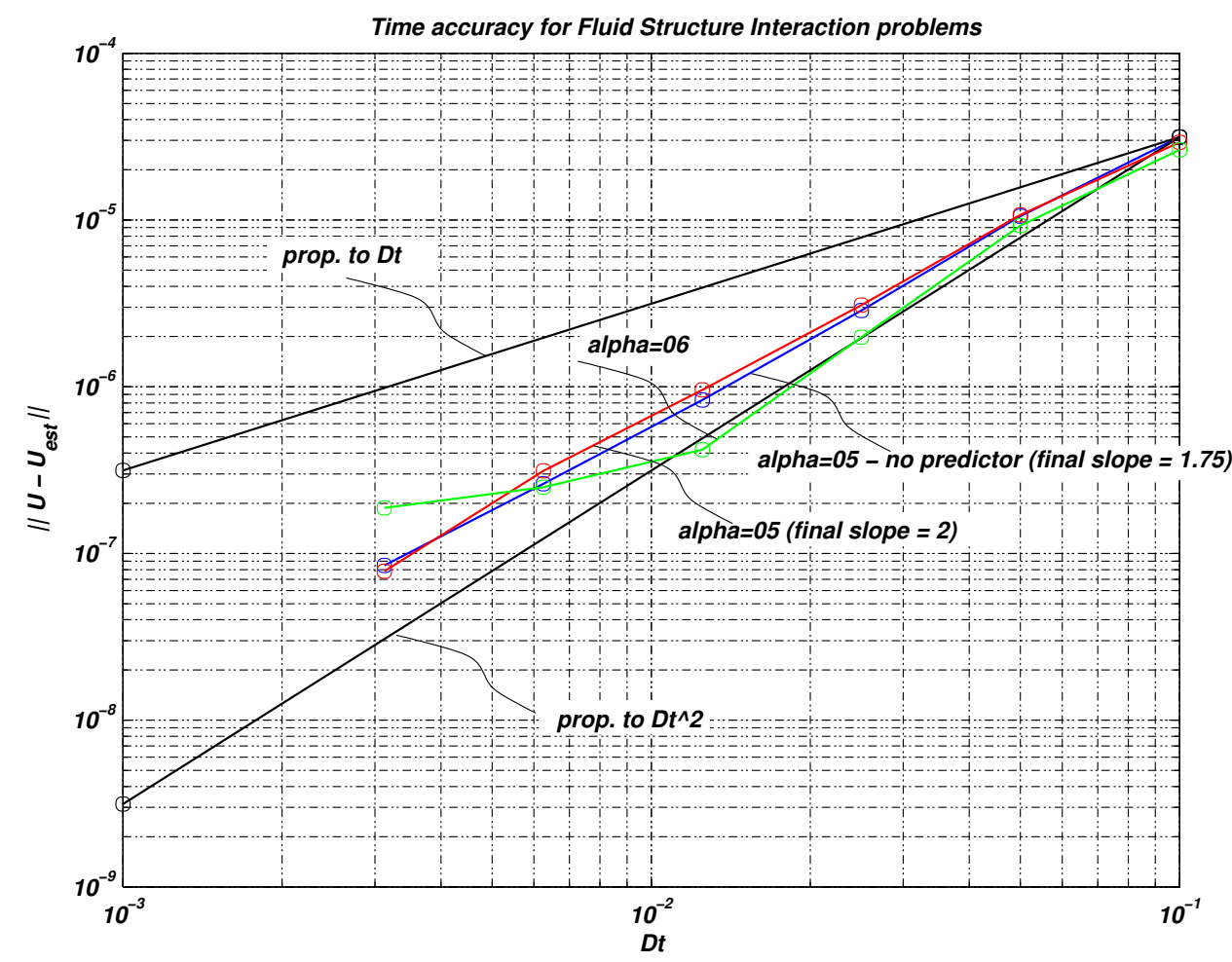

Fig. 14. Experimentally determined order of convergence with $\Delta t$ for the uncoupled algorithm with fourth order predictor.

parameters that have been modified and the dimensionless parameters that may be obtained with them are included.

$$
\begin{aligned}
U_{\infty} & =\mathrm{M}_{\infty}=2 \\
\mathrm{t} & =0.06 \\
\nu & =0.33 \\
m & =0.002 \\
E & =39.6 \\
D & =8.010^{-4} \\
N_{T} & =\frac{D}{m L^{2} U_{\infty}^{2}}=0.025 \\
N_{M} & =\frac{\rho_{\infty} L}{m}=1000.0
\end{aligned}
$$

Therefore according to the section $\S 3.2 .5$

$$
\frac{N_{M}}{N_{T} \mathrm{M}_{\infty}{ }^{2}}=10000>300
$$

implies that the flow is inside the flutter region. 


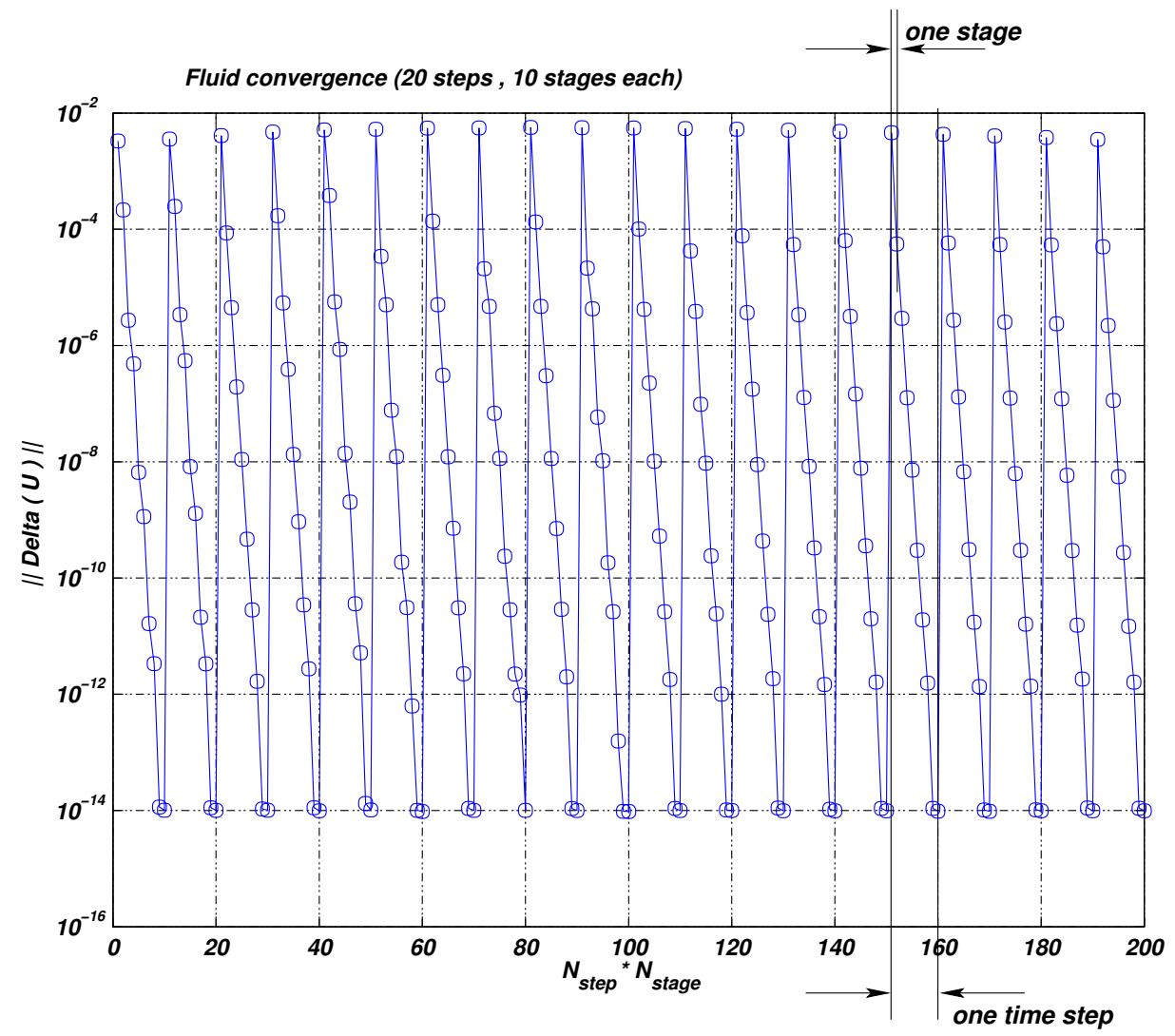

Fig. 15. Convergence of fluid state in stage loop.

The following figures shows results obtained with both strategies, the staged and non-staged algorithms. In order to compare both results in terms of the computational cost, for the nonstaged algorithmic, a time step reduced by the number of stages used for the staged algorithm. So, the cost is similar for each one of them.

The vertical displacements on some points of the plate for the staged algorithm using $n_{\text {stage }}=5$ after approximately 1300 time steps are shown in figure (17). The results for the non-staged algorithm diverge at 40 time steps and are shown in figure (18).

Even though the staged algorithmic shows an extra stability compared with the non-staged one, the conclusions about this numerical experiment are not obvious because the flow regime is in a flutter condition. Further work needs to be done towards the understanding about how the staged algorithmic improves the stability of the whole coupled problem. 


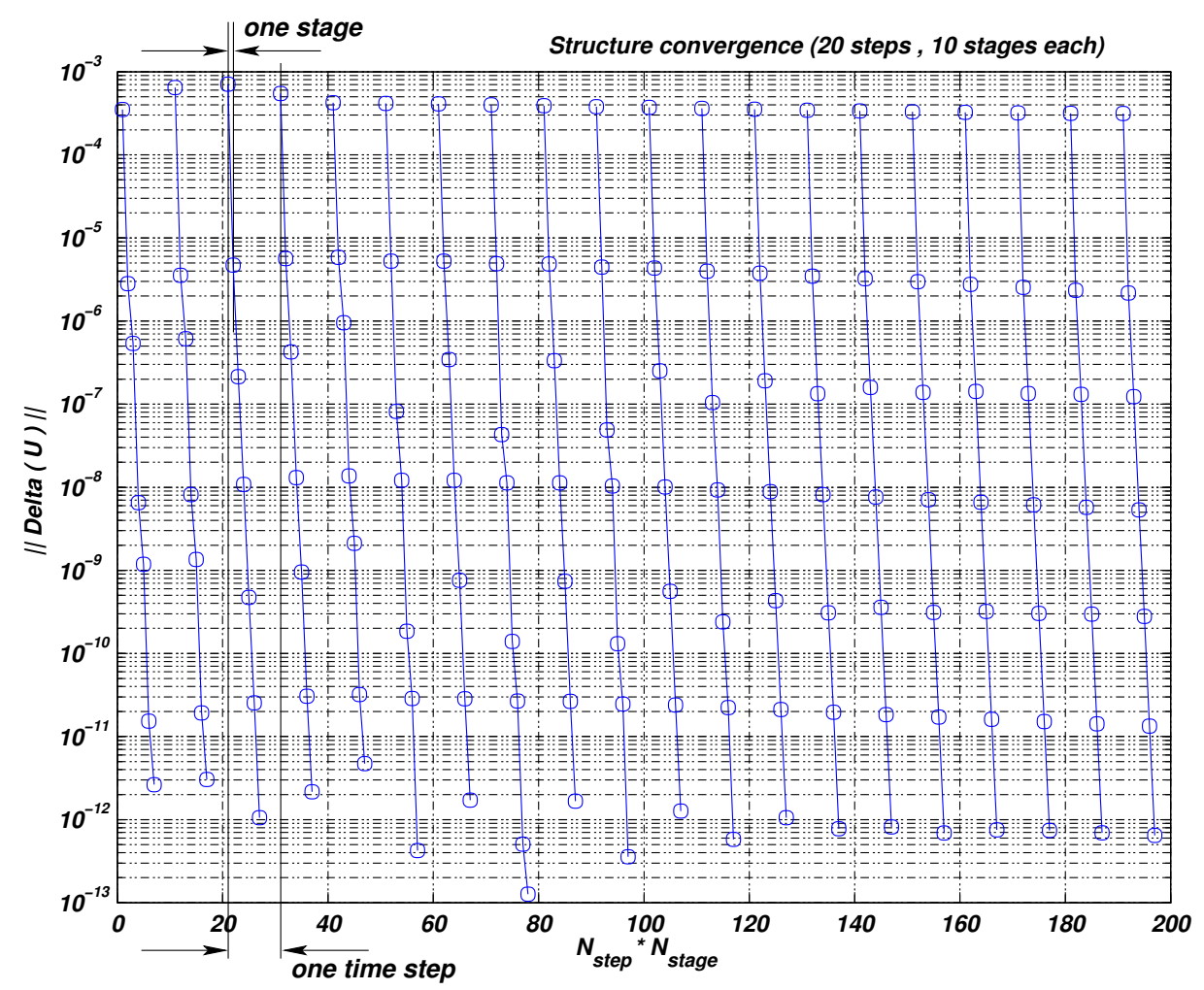

Fig. 16. Convergence of structure state in stage loop.

\section{STABILITY OF THE WEAK/STRONG STAGED COUPLING OUTSIDE THE FLUT- TER REGION}

This section describes the stability properties of the weak coupling when the free stream conditions and plate parameters are such that the oscillations due to flutter do not appear. The flutter region can be characterized by the dimensionless number $F L=N_{M} / N_{T} M_{\infty}^{2}$ (see the previous section). Therefore, in order to study the stability behavior of the weak/strong algorithms ( $n_{\text {stage }}=1$ and $n_{\text {stage }}>1$, respectively) intrinsic to the physical coupling, the region $F L \ll 200$ is studied, particularly $F L=12$ is chosen. This non-dimensional number does not depend on the plate density $m$ then a sweep can be on this variable in a wide range avoiding triggering flutter. The idea is to find a value for this variable in which the weak coupling algorithms becomes unstable while the strong coupling and the uncoupled problems remain stable (i.e., fluid pressures are not transferred to the structure). To accomplish convergence in the nonlinear loop for the fluid problem 2 Newton iterations (typically for this problem, 3-5 orders of magnitude are lowered in the residual in 2 Newton loops) are adopted. The mesh is the same as in the previous simulations and the Courant number is $C F L=0.5$. The plate mass varies in the range $m=35$ and $m=0.0001$. It was found that the value of $m$ where instabilities appear relies in the neighbor of $m=0.65$. Above this value the weakly coupling scheme is stable, below this, 


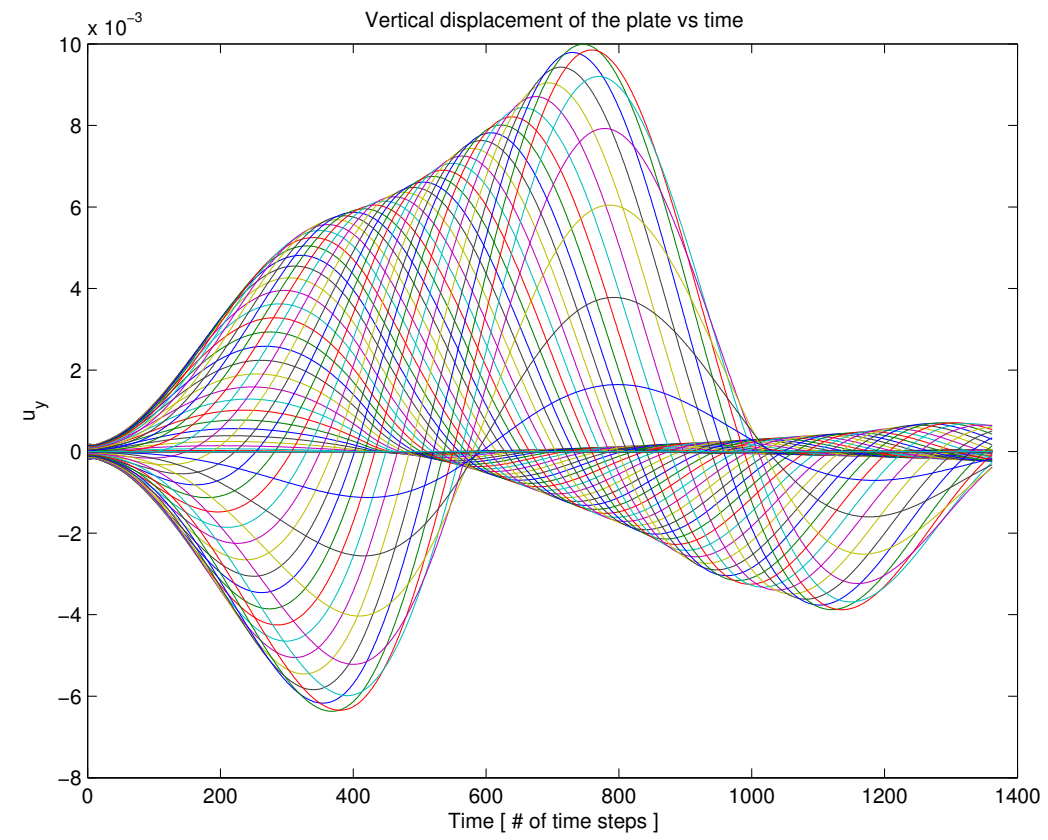

Fig. 17. Stability analysis - Staged algorithm with $n_{\text {stage }}=5$. Vertical displacements of the plate vs time

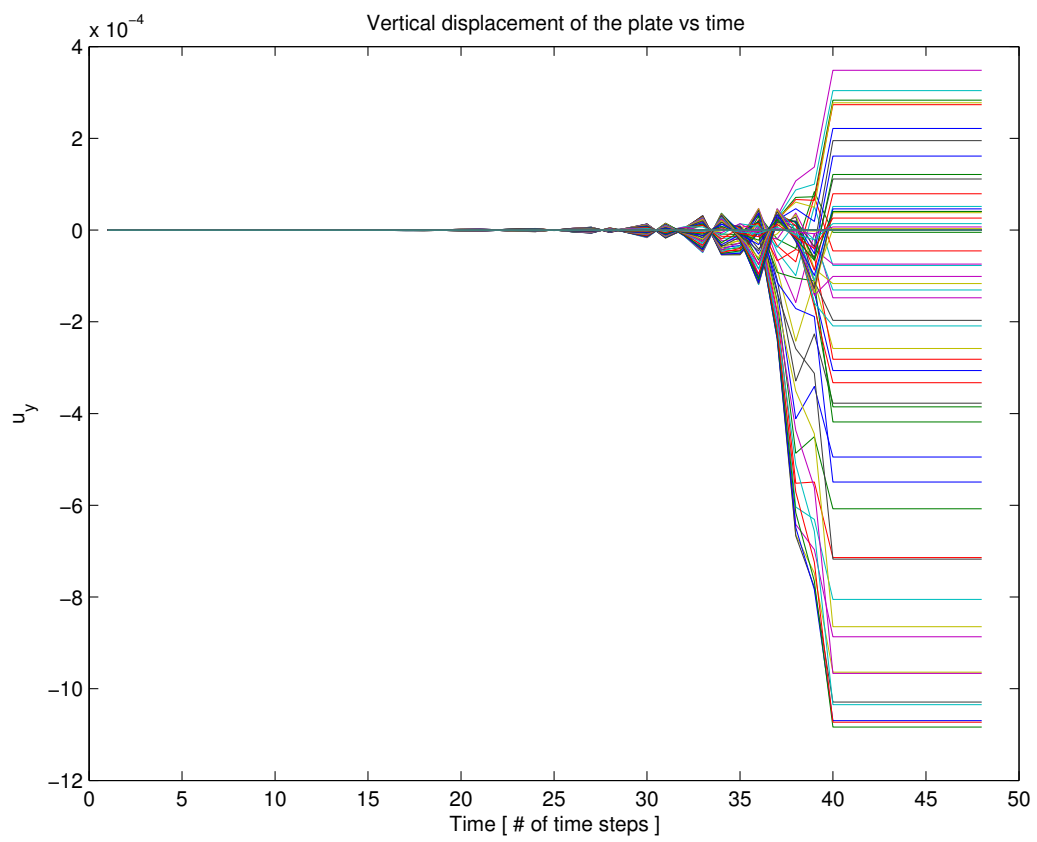

Fig. 18. Stability analysis - Non-staged algorithm. Vertical displacements of the plate vs time

the weakly coupling algorithm is unstable meanwhile each sub-problem (fluid and structure) are stable when considering no coupling. Instabilities disappear when the strong coupling scheme with $n_{\text {stage }}=2$ is considered. Moreover, even when a lower value of $m$ is used, i.e. $m=0.0135$ 
and $C F L=1$, only 2 stages are enough to achieve convergence of the strong coupling algorithm. Obviously, at this point each detached problem is still stable (see figures (19) and (20)). In the case of $m=0.0001$ a smaller $C F L$ number is needed (e.g. $C F L=0.5$ ) in order to have at least 15 time steps in one period (recall that the plate frequency depends on the plate density).

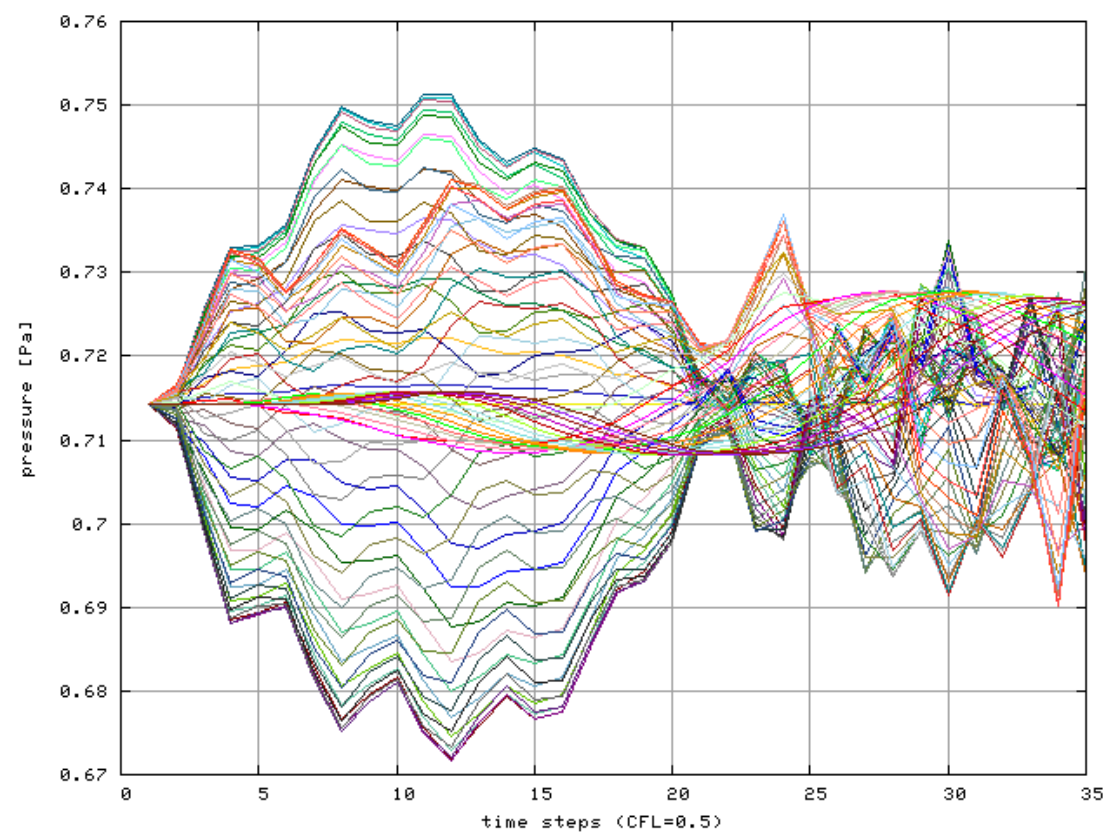

Fig. 19. Unstable weak coupling for $m=0.0135$ and $C F L=0.5$

Even though the convergence of the coupled problem is not affected when considering a very small plate density and a strong partitioned scheme, it is necessary to refine the fluid mesh in the normal direction to the plate in order to have a better definition of the problem in that direction, i.e. a wave train is propagated in the fluid with the plate frequency (recall that the plate frequency grows as the plate density decrease, $\left.\omega_{\mathrm{str}}=2 \pi / T_{\mathrm{str}}=\left[m L^{4} / D\right]^{-1 / 2}\right)$. In the coupled simulation the interaction may produce the amplification of the pressures and plate displacements. This fact can be shown in figures (21) and (22). Figure (21) shows the plate deflection when $m=0.00135$ and mesh size of the fluid mesh near the plate is $h_{y}=0.018808 \mathrm{~m}$ and $h_{x}=0.035625 \mathrm{~m}$. Figure (22) shows the same results when considering an homogeneous refinement (i.e., $h_{y}=0.00932 \mathrm{~m}$ and $h_{x}=0.01781 \mathrm{~m}$ ). The coarse mesh exhibit a spurious vibration mode similar to a flutter mode that is corrected in the finer mesh.

\section{CONCLUSIONS}

Stability is enhanced through a strong coupling scheme and it shows to be necessary for situations where the structural response is fast. Partitioned schemes using staged strong coupling 


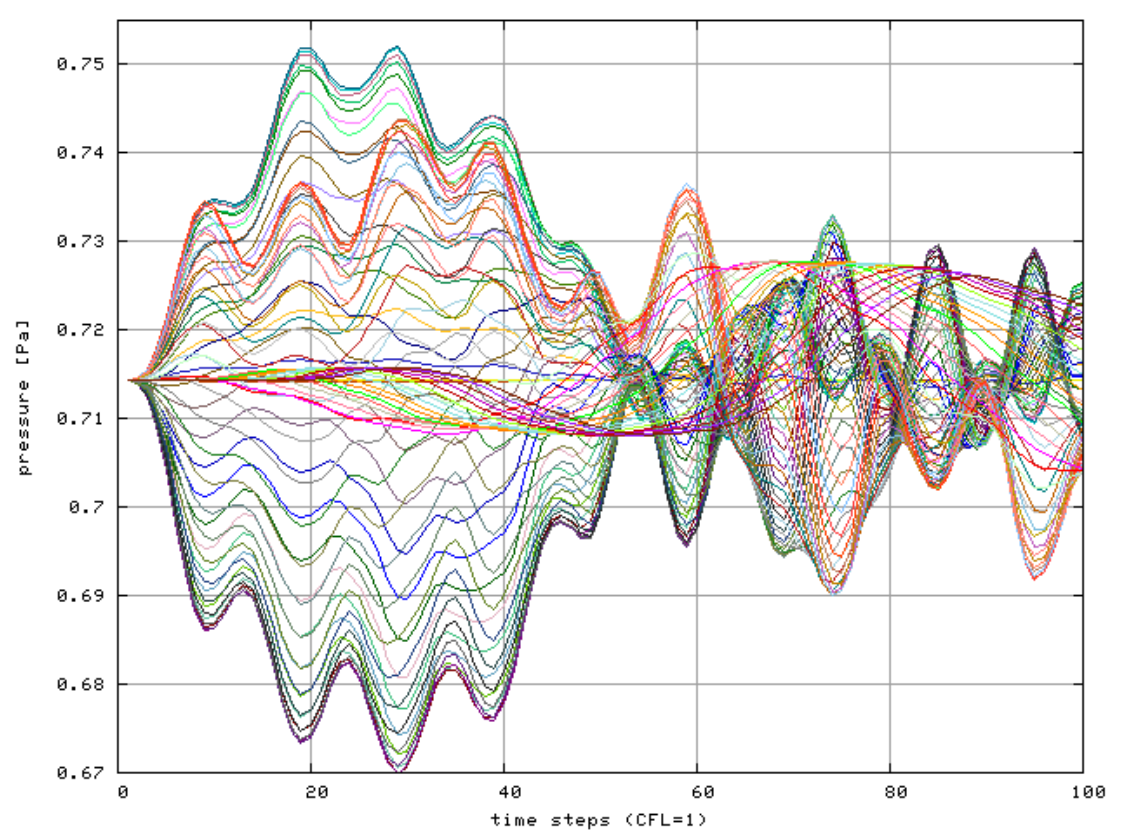

Fig. 20. Stable staged coupling for $m=0.0135, C F L=1$ and $n_{\text {stage }}=2$

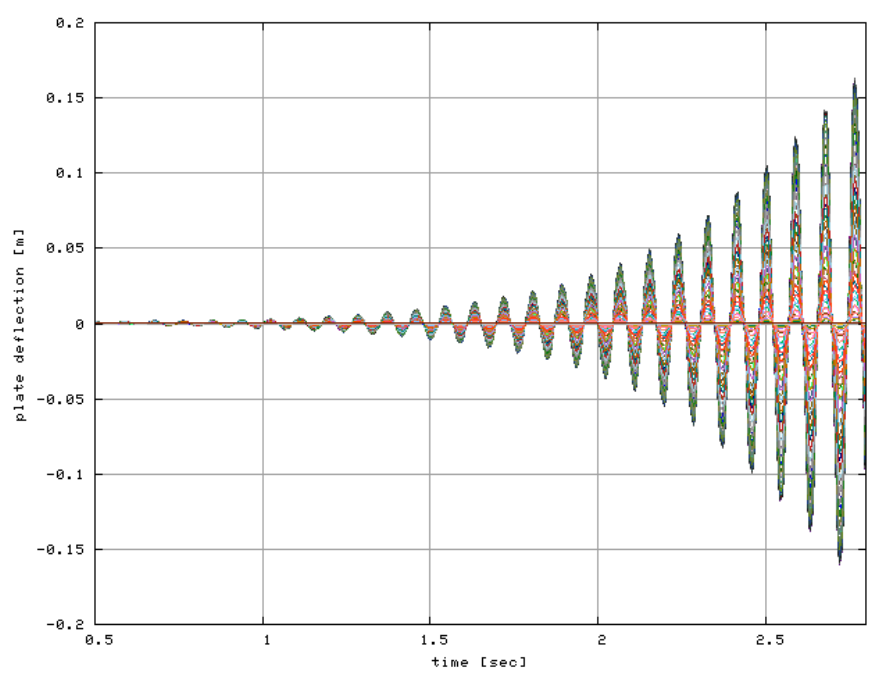

Fig. 21. Strong partitioned scheme in a coarse mesh

shows to be very efficient avoiding the tedious and problem dependent task of building a monolithic coupling formulation. For the benchmark considered in this work two stages were enough for having the same behavior of the monolithic scheme. Furthermore, the staged strategy provides a smooth blending between weak coupling and strong coupling, i.e. moderately coupled problems that can not be treated with the pure weak coupling approach, can be solved with the staged algorithm using few stages per time step. 


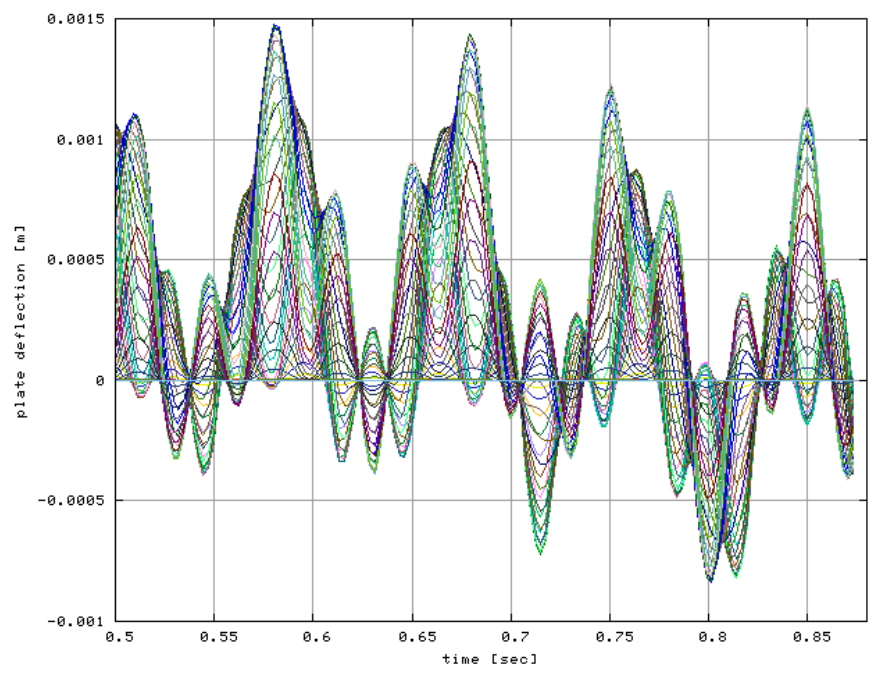

Fig. 22. Strong partitioned scheme in a fine mesh

Time-accuracy shows to be in agreement with the accuracy of the underlying fluid and structure solvers, if an accurate enough predictor is used. Second order accuracy can be obtained with second order fluid and structure solvers, and one stage coupling with a high order predictor, as already reported in Reference [2].

The elastic flat plate problem is geometrically simple, but gives physical insight in the flutter phenomena, and was very useful in testing the proposed algorithm in a wide range of nondimensional parameters.

\section{Acknowledgements}

This work has received financial support from Consejo Nacional de Investigaciones Científicas y Técnicas (CONICET, Argentina, grants PIP-5271/2005), Universidad Nacional del Litoral (Argentina, grants CAI+D 2005-10-64) and ANPCyT (Argentina, grants PICT 12-14573/2003 and PME 209/2003). Extensive use of freely distributed software such as GNU/Linux OS, MPICH, PETSc, Metis, Octave, OpenDX and many others is done in this work.

\section{References}

[1] V. Gnesin and R. Rzadkowski. A coupled fluid structure analysis for 3-d inviscid flutter of IV standard configuration. Jornal of Sound and Vibration., 49:349-369, 2005. 
[2] R. Piperno and C. Farhat. Partitioned procedures for the transient solution of coupled aeroelastic problems. Part II: energy transfer analysis and three-dimensional applications. Computer Methods in Applied Mechanics and Engineering, 190:3147-3170, 2001.

[3] E. Lefrancois. Numerical validation of a stability model for a flexible over-expanded rocket nozzle. International Journal for Numerical Methods in Fluids., 49:349-369, 2005.

[4] E. Dowell, E. Crawley, H Curtiss, D. Peters, R. Scanlan, and F. Sisto. A Modern Course in Aeroelasticity. Kluwer Academic Publishers, Dordrecht, 1995.

[5] C.A. Felippa, K.C. Park, and C. Farhat. Partitioned analysis of coupled mechanical systems. Computer Methods in Applied Mechanics and Engineering, 190:3247-3270, 2001.

[6] K. C. Park and C. A. Felippa. A variational principle for the formulation of partitioned structural systems. International Journal for Numerical Methods in Engineering., 47:395-418, 2000.

[7] W. Dettmer and D. Peric. A computational framework for fluid-rigid body interaction: Finite element formulation and applications. Computer Methods in Applied Mechanics and Engineering, 195:1633-1666, 2006.

[8] E. Lopez, N. Nigro, M. Storti, and J. Toth. A minimal element distortion strategy for computational mesh dynamics. International Journal for Numerical Methods in Engineering., 2006.

[9] M. Senga T. Tezduyar. Determination of the shock-capturing parameters in supg formulation of compressible flows. In Tsinghua University Press \& Springler-Verlag, editor, Computational Mechanics WCCM IV, Beijing, China. 2004, 2004.

[10] J.C. Houbolt. A study of several aerothermoelastic problems of aircraft structures. Mitteilung aus dem Institut fur Flugzeugstatik und Leichtbau 5, E.T.H., Zurich, Switzerland, 1958. 